\title{
Forum
}

\section{Schwerpunktthema: Chinas Grenzen}

Die Bedeutung Chinas wächst seit Jahrzehnten unaufhaltsam. Galt die Volksrepublik lange Zeit als aufstrebende Regionalmacht, ist sie über dieses Stadium längst hinausgetreten. China - so die weitläufige Einschätzung - wird in der nahen Zukunft der einzige wirkliche weltpolitische Gegenspieler der Vereinigten Staaten sein. Die Gründe hierfür sind vielfältig. Zu nennen sind etwa die Wirtschaftskraft und die enormen Zuwachsraten der chinesischen Volkswirtschaft, die regelmäßig weit über denen der meisten OECD-Staaten liegen und das Riesenreich mit seinen mehr als 1,3 Milliarden Einwohnern zu einem „Big Player“ der Weltwirtschaft machen. Aber auch seine militärische Stärke ist Ausdruck dieser Entwicklung. Es scheint, als könnte nichts, als könnten keine Grenzen den Aufstieg Chinas stoppen.

Doch ein genauerer Blick offenbart vielfältige Probleme und Fehlentwicklungen: etwa die Rückständigkeit vieler ländlicher Gebiete, die teilweise horrende Umweltverschmutzung, die demografischen Auswirkungen der Ein-Kind-Politik der letzten Jahrzehnte und nicht zuletzt die Demokratiefrage. Darüber hinaus ist noch nicht gänzlich absehbar, in welche Richtung sich die Volksrepublik nach den jüngsten Wechseln an der Spitze von Staat und Kommunistischer Partei politisch, gesellschaftlich, wirtschaftlich oder auf internationalem Terrain entwickeln wird.

Vor diesem Hintergrund beleuchtet das folgende Forum die Grenzen Chinas in seiner weiteren Entwicklung aus dem Blickwinkel unterschiedlicher Teildisziplinen der Politikwissenschaft. Hierfür beschäftigen sich Sebastian Heilmann und Dirk Schmidt aus theoretischer Perspektive mit den Grenzen des Wachstums Chinas in ökonomischer Hinsicht. Thomas Heberer fragt aus dem Blickwinkel der Politischen Systemforschung nach den Grenzen der Freiheit im Modernisierungsprozess der Volksrepublik. Xuewu Gu analysiert schließlich aus der Perspektive der Internationalen Beziehungen die Grenzen der Sicherheit Chinas. 